\title{
2.4GHZ Class F Power Amplifier for Wireless Medical Sensor Network
}

\author{
Wei Cai ${ }^{1}$, Jie Gong ${ }^{2}$, NanSong $\mathrm{Wu}^{3}$ \\ ${ }^{1}$ Department of Electrical Engineering and Computer Science, University of California \\ Irvine, CA, USA \\ caiw2@uci.edu \\ ${ }^{2}$ School of Mechanical Engineering \\ Georgia Institute of Technology, Atlanta, GA, USA \\ jgong6@gatech.edu \\ ${ }^{3}$ Department of Electrical and Computer Engineering, Florida International University \\ Miami, FL, USA \\ nwu@fiu.edu
}

\begin{abstract}
The objective of this research was to design a $2.4 \mathrm{GHz}$ class F Power Amplifier (PA), with 0.18um Semiconductor Manufacturing International Corporation (SMIC) CMOS technology by using Cadence software, for sensor network, especially for health care. The ultimate goal for such application is to minimize the trade-offs between performance and cost, and between performance and low power consumption design. This power amplifier can transmit $18 \mathrm{dBm}$ output power to a $50 \Omega$ load. The power added efficiency is $46 \%$ at $1 \mathrm{~dB}$ compression point and the power gain is $18 \mathrm{~dB}$, the total power consumption is $2.8 \mathrm{~W}$. The performance of the power amplifier meets the specification requirements of the desired.
\end{abstract}

Keywords: two stage, class F, cascade, power amplifier

\section{Introduction}

An emerging application in nanotechnology is the field of nano-electronics. The field of nano-electronics leverages new methods and materials to build electronic devices with feature sizes on the nanoscale [1]. Complementary metaloxide-semiconductor (CMOS) is mature technology that has a long history in integrated circuit fabrication. CMOS technology has been used to implement a vast array of devices critical to modern society. Some of the most ubiquitous applications include devices such as microprocessors, microcontrollers, static RAM, image sensors (CMOS sensor), data converters, and highly integrated transceivers for many types of communication applications.

The drive to miniaturize such devices has refined CMOS into a capable technology for fabricating nanotechnology devices. In the current technology, features less that $10 \mathrm{~nm}$ across can be made. With these techniques and materials, billions of transistors can be fabricated on a wafer - allowing a vast array of functons to be integrated on a single chip. These qualities make CMOS is a strong process for fabricating nanoscale devices. There are some physical limitations to silicon-based processes, however, and there is room for improvement in the CMOS process for nano-fabrication applications.

Wireless sensor networks (WSN) are collections of distributed sensors which provide data about their environment. This data can relate to many applications, such as human body monitoring or environmental conditions such as temperature, soil moisture, wind speed, or sound levels. Nodes in this network, after collecting data can then pass the collected data through the network to a central location. Contemporary networks are often bidirectional which enables the control of sensor activity, as well. Such networks are widely used in industrial and consumer applications, providing process monitoring and control, healthcare monitoring, and so on.

The basic architecture of a WSN is shown in figure 1. A WSN is composed of a few to several hundreds or even thousands nodes, where each node is connected to one or more sensors. A sensor network node typically has several parts: a radio transceiver with an internal or an external antenna, a microcontroller, and a battery or energy harvesting mechanism to provide energy. 
Wireless medical sensor networks have offered significant improvements to the healthcare industry in the 21 st century. Devices are arranged on a patient's body and can be used to closely monitor the physiological condition of patients. These medical sensors monitor the patient's vital body parameters, such as temperature, heart rate, blood pressure, oxygen saturation, and transmit the data to a doctor in real time [1]. When a doctor reviews the transmitted sensor readings, they can get a better understanding of a patient's health conditions. People living in rural areas would especially benefit, since $9 \%$ of physicians work in rural areas while almost $20 \%$ of the US population lives there [2]. A shortage of physicians and specialist is a big issue in such areas, even today. But Wireless Medical Sensor Network technology has the potential to alleviate the problem.

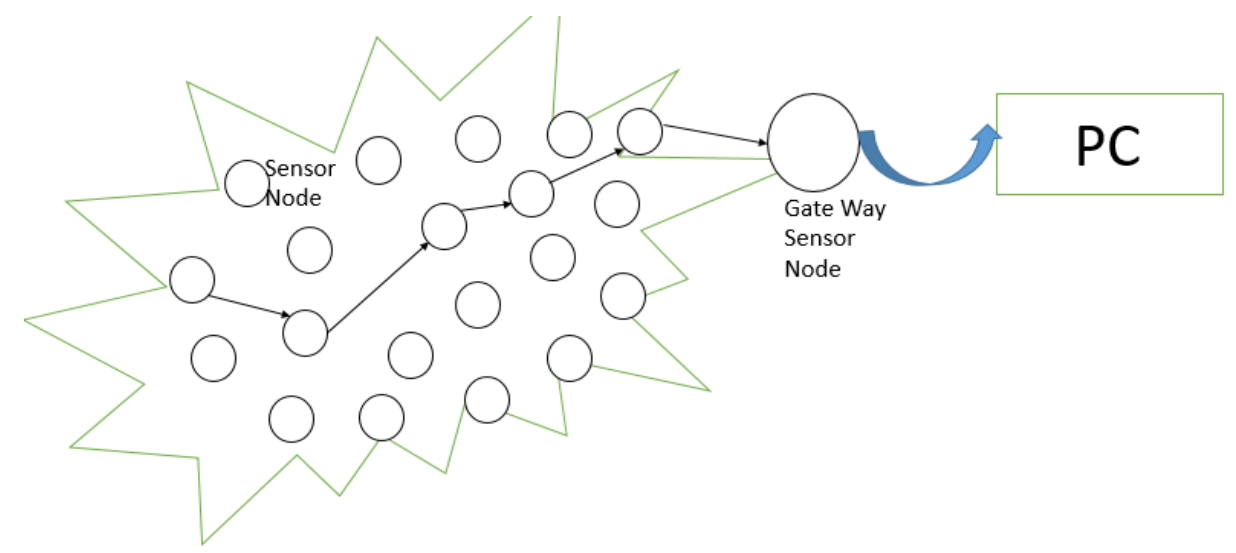

Fig. 1: Basic structure of a typical sensor node.

Wireless medical sensor networks have offered significant improvements to the healthcare industry in the 21 st century. Devices are arranged on a patient's body and can be used to closely monitor the physiological condition of patients. These medical sensors monitor the patient's vital body parameters, such as temperature, heart rate, blood pressure, oxygen saturation, and transmit the data to a doctor in real time [1]. When a doctor reviews the transmitted sensor readings, they can get a better understanding of a patient's health conditions. People living in rural areas would especially benefit, since $9 \%$ of physicians work in rural areas while almost $20 \%$ of the US population lives there [2]. A shortage of physicians and specialist is a big issue in such areas, even today. But Wireless Medical Sensor Network technology has the potential to alleviate the problem.

\section{Background Introduction}

Fig.2 shows that the basic sensing node can collect the physiological signals (e.g.: such as EEG, ECG, body temperature, blood pressure, heart beat etc.), when attached to a human body [2]. The processing unit processes all the sensed signals, then sends out the data based on communication protocols [3] [4] [5] [6]. All the processed data will be transmitted through a wireless link to a portable, personal base-station. Doctors can then obtain all the patients' data through the network. Two major requirements for a WSN are the need for a compact form factor and low cost. As seen in the figure 3, the power amplifier is the major consumer of power in a sensor node. A power amplifier design for a WSN would ideally be low cost while providing high gain, high linearity and low power consumption. This is a challenging design requirement. For instance, while silicon designs are low cost, amplifiers built in silicon do not typically provide high gain or linearity. Other LNA, Mixer and VCO talked at [7].

The rapid growth of wireless systems has created increasing demand of lower cost, smaller form factor systems with superior and complex functionalities. While III/V semiconductors, such as GaAs and SiGe, can easily achieve high power output and high gain, they incur high costs. Often to reduce cost, circuits designed are targeted for the CMOS process, since CMOS is a common and well-established process. For medical devices, the power amplifier is the main power consumer. For RF power amplifiers design, there is always a trade of PAE, power output and the supply voltage for different standards and applications. And for this proposal, a class $\mathrm{F}$ power amplifier is the main design under investigation. In order to meet the standards, the PA is designed as shown in table 1 . 


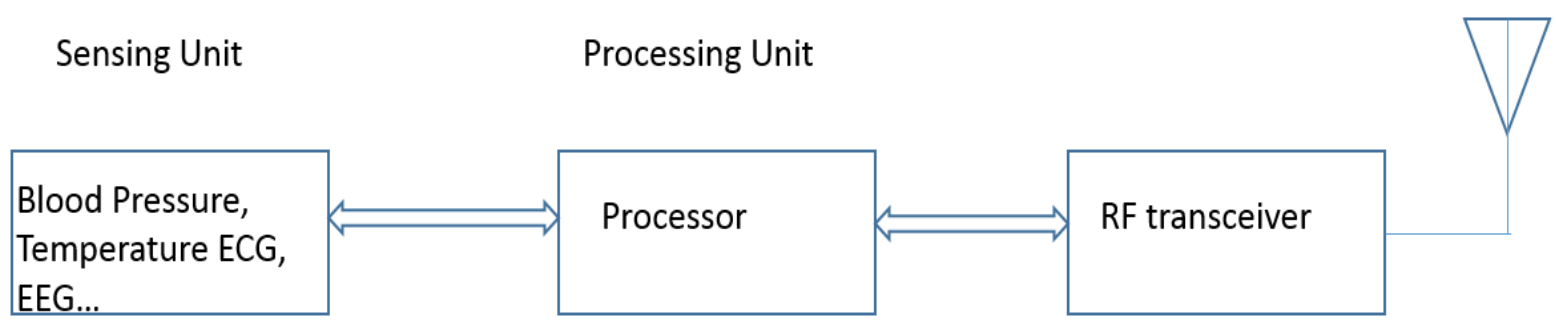

Fig. 2: Block diagram of a typical WSN.

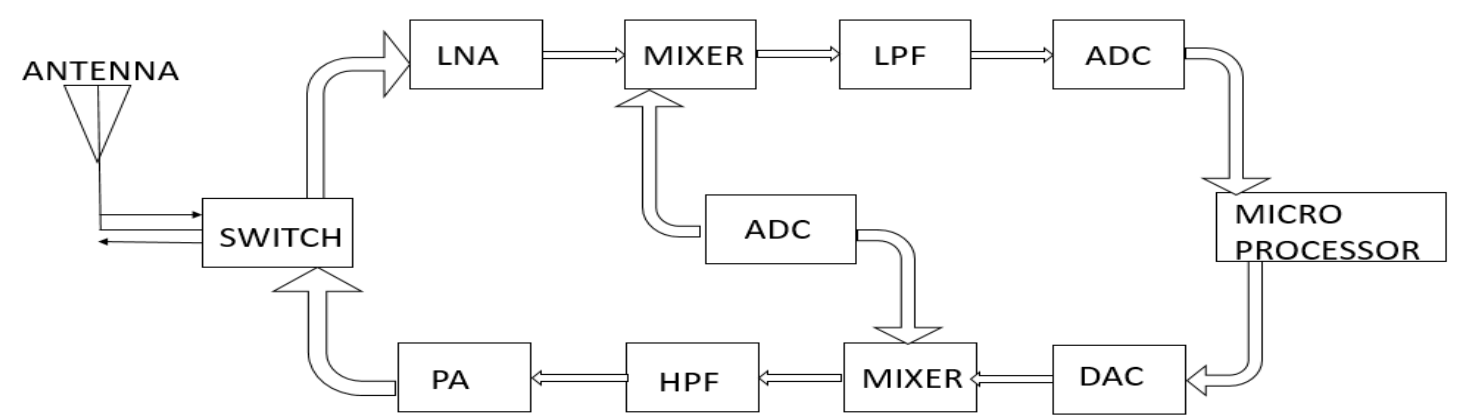

Fig. 3: Block diagram of a transmitter.

Table 1 : PA design requirement.

\begin{tabular}{|l|l|}
\hline Parameter & Regular \\
\hline Output Power & $18 \mathrm{dBm}$ \\
\hline PAE & $30 \%$ \\
\hline Stability & $>1$ \\
\hline S11 & $-10 \mathrm{~dB}$ \\
\hline
\end{tabular}

\section{PA Design}

Over the past 30 years, research on CMOS radio-frequency (RF) front-end circuits has progressed extremely quickly. The ultimate goal for the wireless industry is to minimize the trade-offs between performance and cost, and between performance and low power consumption design [8] [9][10]. The proposed Class $\mathrm{F}$ amplifier has low output power and good linearity based on the IEEE $802.11 \mathrm{~b}$ communication protocol [9]. The $2.4 \mathrm{GHz}$ class $\mathrm{F}$ PA is a two stage common-source amplifier. The first stage is a driver stage, used for providing sufficient driving capability and a proper gain, as seen in figure 4(a). And the second stage is the power output stage which used for performing sufficient output power, as seen in figure $4(b)[11]$.

\subsection{Class F PA Design}

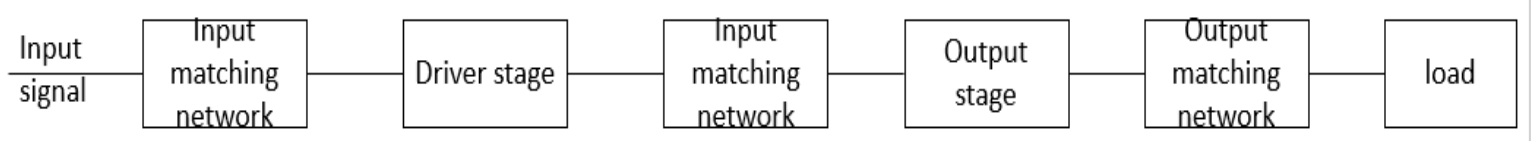

Fig. 4: Block diagram of a class F power amplifier.

Power amplifiers are critical components in daily life. One major application that touches many people's lives is modern communication systems, such as in our cell phones. Recently, research in power amplifier technologies is 
becoming more and more common, due to this far-reaching impact. This research is focused on how to design power amplifiers with low power, high linearity, broadband, high power added efficiency (PAE) and low cost. In the case of Class $\mathrm{AB}$ power amplifiers, there is a fundamental tradeoff between linearity and efficiency [11]. For class $\mathrm{F}$ power amplifier, the main advantage is high performance and low power consumption. Furthermore, it has less power losses for the same frequency compare to other types of power amplifier. The class F doesn't have many components, so it makes implement in the silicon cheaper and make the cost cheaper.

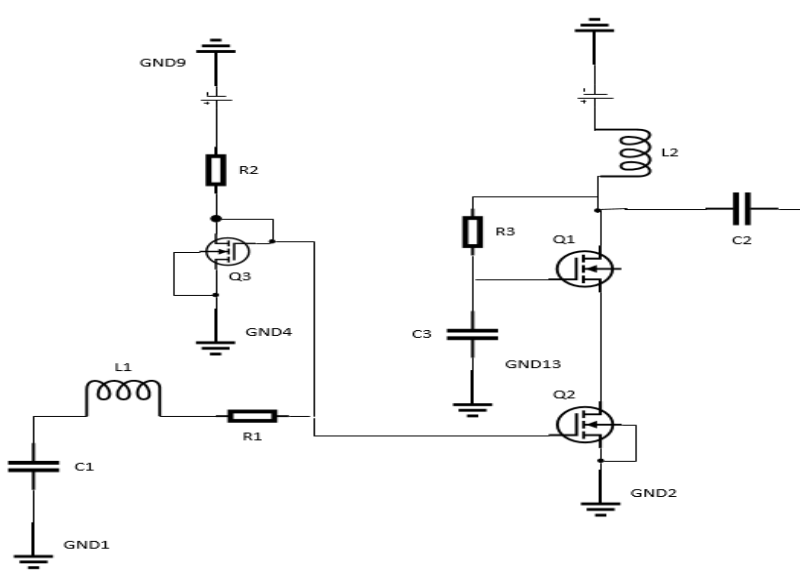

(a)

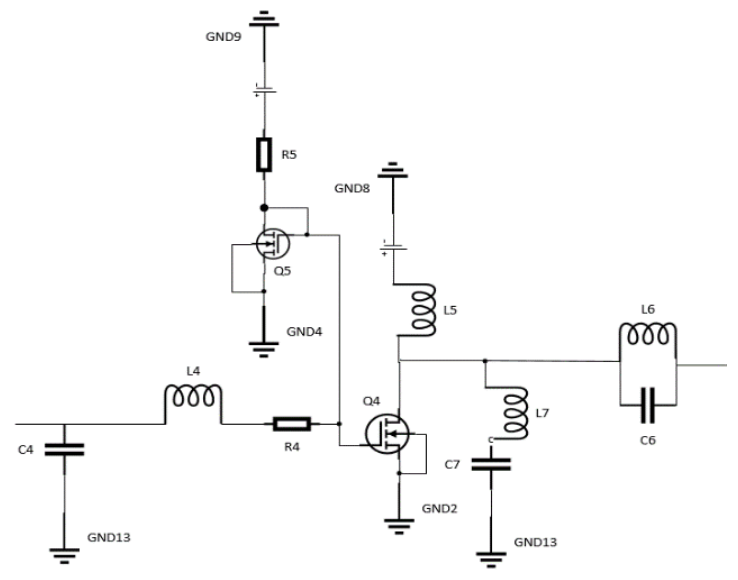

(b)

Fig. 5: (a) Schematic of driver stage schematic (b) Schematic of output stage schematic.

The drive stage as seen in figure 5 (a), is a cascode structure [12]. Cascode structures are a common approach to mitigate the low gain typically seen in silicon circuits. Cascode structure provide higher gain. Transistor M1 is self-biased, which reduces headroom. The component values can be seen in the Table 2.

Table 2: 2.4GHz PA driver stage component.

\begin{tabular}{|l|l|}
\hline Parameter & Size (Unit) \\
\hline Q1 & W/L=0.6um/0.6um $(\mathrm{f}=56, \mathrm{~m}=16)$ \\
\hline Q2 & W/L=0.3um/0.6um $(\mathrm{f}=88, \mathrm{~m}=36)$ \\
\hline Q3 & W/L=0.3um/0.6um $(\mathrm{f}=2, \mathrm{~m}=6)$ \\
\hline R1 & 14.5 Ohm \\
\hline R2 & $10 \mathrm{~K} \mathrm{Ohm}$ \\
\hline R3 & $80 \mathrm{Ohm}$ \\
\hline $\mathrm{L} 1$ & $36 \mathrm{nH}(\mathrm{Q}=20)$ \\
\hline $\mathrm{L} 2$ & $15 \mathrm{nH}(\mathrm{Q}=20)$ \\
\hline C1 & $240 \mathrm{fF}$ \\
\hline C2 & $10 \mathrm{pF}$ \\
\hline C3 & $100 \mathrm{pF}$ \\
\hline
\end{tabular}

The output stage is shown in figure 5 (b). In most cases, Class F power amplifiers are usually biased as Class AB or Class B states, thus eliminating the harmonics, providing a square wave shape at the output. More specifically, the odd order harmonic impedances are infinite (besides the fundamental order), and the even order harmonic impedances are zero. So for the circuit, at the output port, the second harmonic impedance should be zero and the third harmonic impedance should be infinite. To achieve this goal, a series resonant circuit and a parallel resonant circuit are designed. In Si CMOS technology, the inductors' Q usually set as 20 for simulation. Due to its low cost, the capacitors and the inductors can be integrated on chip. But at the output stage, the inductors are large, so including them on the die has high area cost. 
After the output stage and driver stage, the inter-stage matching circuit is more challenging. If the input of second stage and output of the first stage are all conjugate matched to $50 \Omega$, the two stages can be connected directly. The complete optimized circuit is shown in Figure 6.

Table 3: 2.4GHz PA output stage component.

\begin{tabular}{|l|l|}
\hline Parameter & Size (Unit) \\
\hline $\mathrm{Q} 4$ & $\mathrm{~W} / \mathrm{L}=0.3 \mathrm{um} / 0.6 \mathrm{um}(\mathrm{f}=66, \mathrm{~m}=24)$ \\
\hline Q5 & W/L=0.3um/0.6um $(\mathrm{f}=4, \mathrm{~m}=2)$ \\
\hline R4 & $5 \mathrm{Ohm}$ \\
\hline R5 & $80 \mathrm{Ohm}$ \\
\hline L4 & $30 \mathrm{nH}(\mathrm{Q}=20)$ \\
\hline L5 & $10 \mathrm{nH}(\mathrm{Q}=20)$ \\
\hline L6 & $10 \mathrm{nH}(\mathrm{Q}=20)$ \\
\hline L7 & $1 \mathrm{nH}(\mathrm{Q}=20)$ \\
\hline C4 & $800 \mathrm{fF}$ \\
\hline C6 & $390 \mathrm{pF}$ \\
\hline C7 & $1.1 \mathrm{pF}$ \\
\hline
\end{tabular}

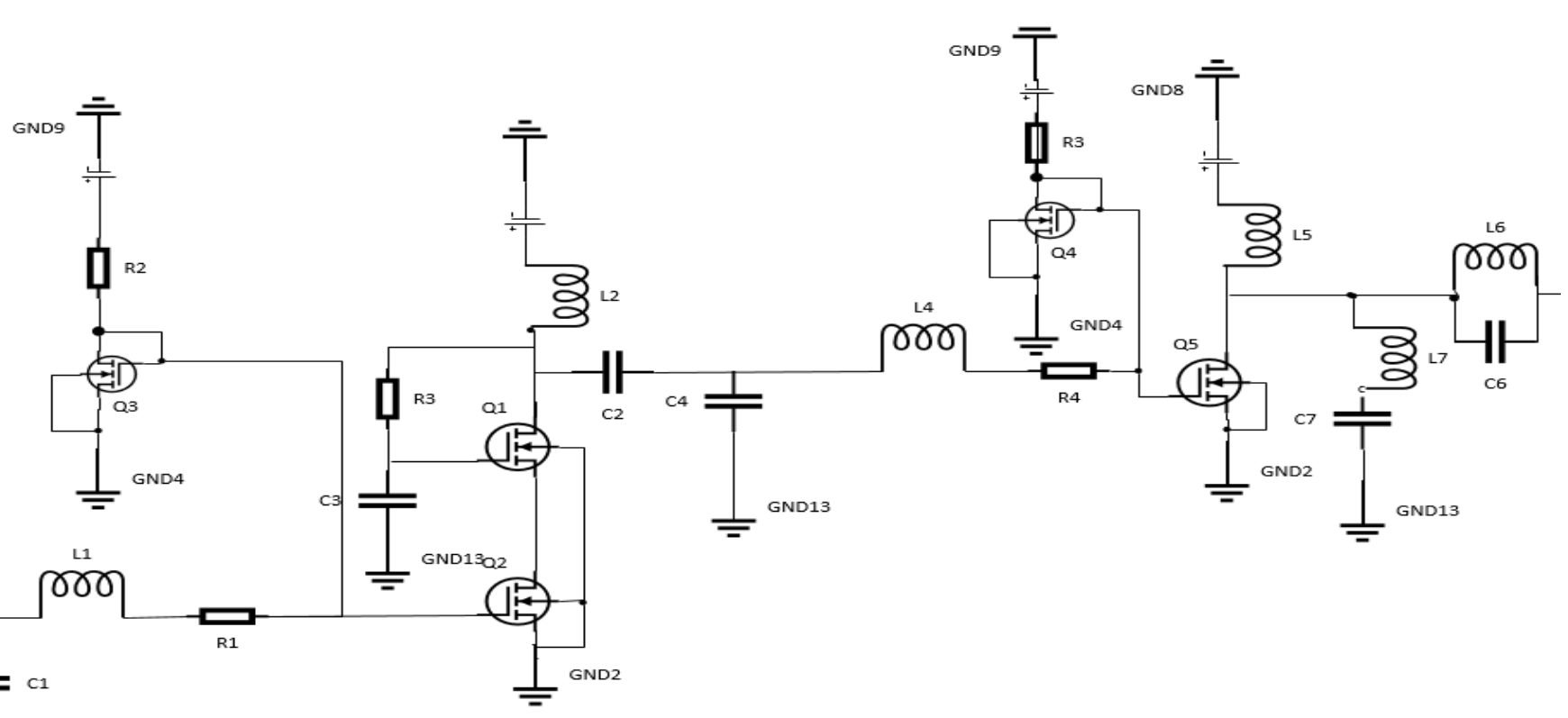

$\underline{\underline{n}}{ }^{\mathrm{GND} 1}$

Fig. 6: Overall circuit schematic.

\subsection{Results}

As seen in figure 7(a), the output power is $10 \mathrm{dBm}$. As seen in figure 6(b), the frequency is at $2.4 \mathrm{GHz}$ the $\mathrm{S} 11$ is less than $-10 \mathrm{~dB}$, also, the total power of the PA is $3.5 \mathrm{~W}$.

As seen in figure 8 (a), $\mathrm{Kf}$ is larger than 1 for all frequencies from 1 to $3 \mathrm{GHz}$, so this circuit is totally stable. And the $\mathrm{PAE}$ is $46 \%$ at input power $0 \mathrm{~dB}$. 

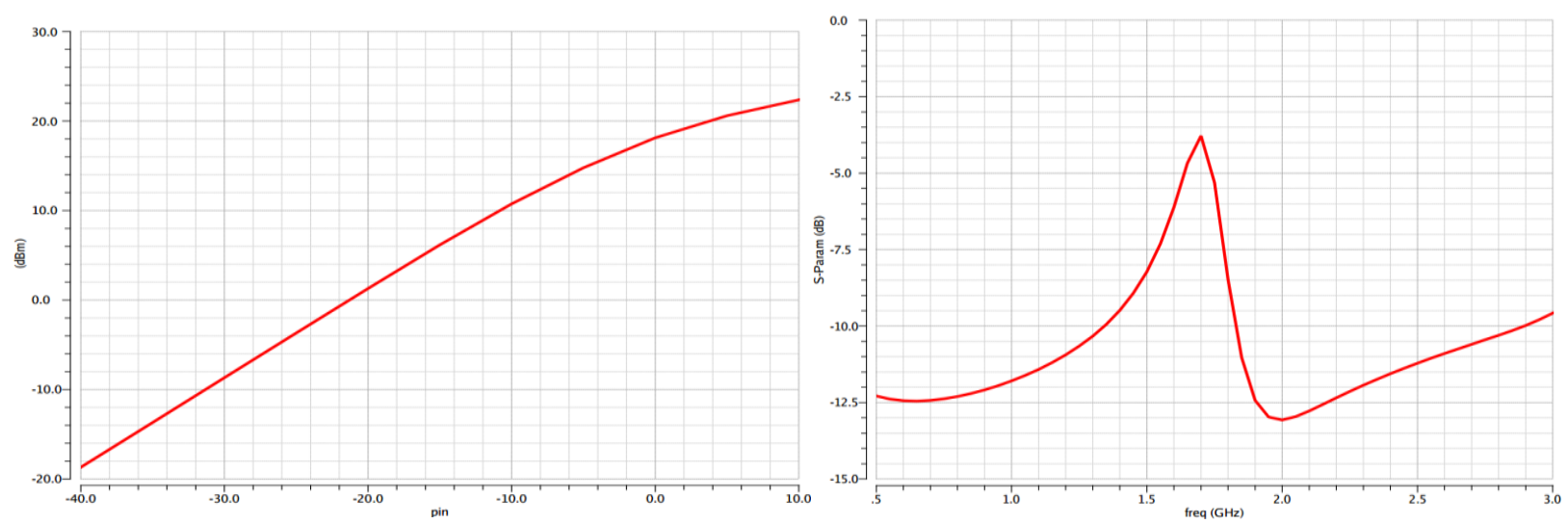

Fig. 7: (a) PA output (b) S11.

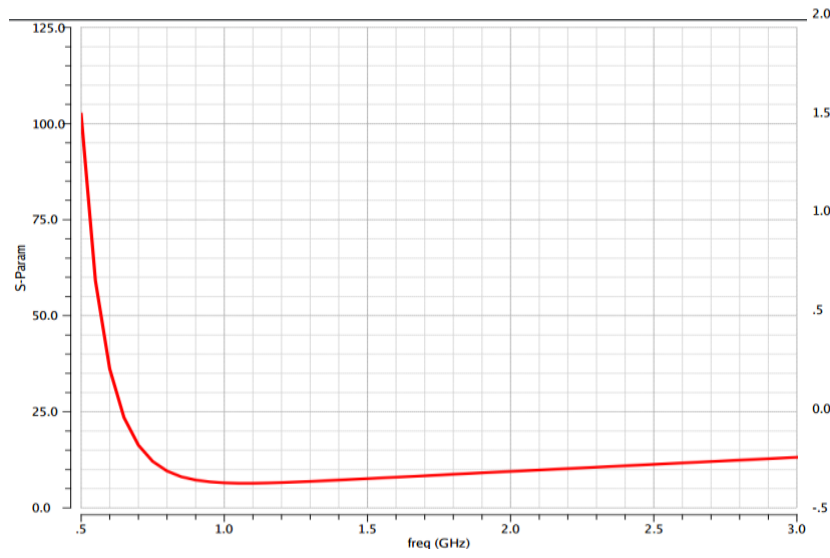

(a)

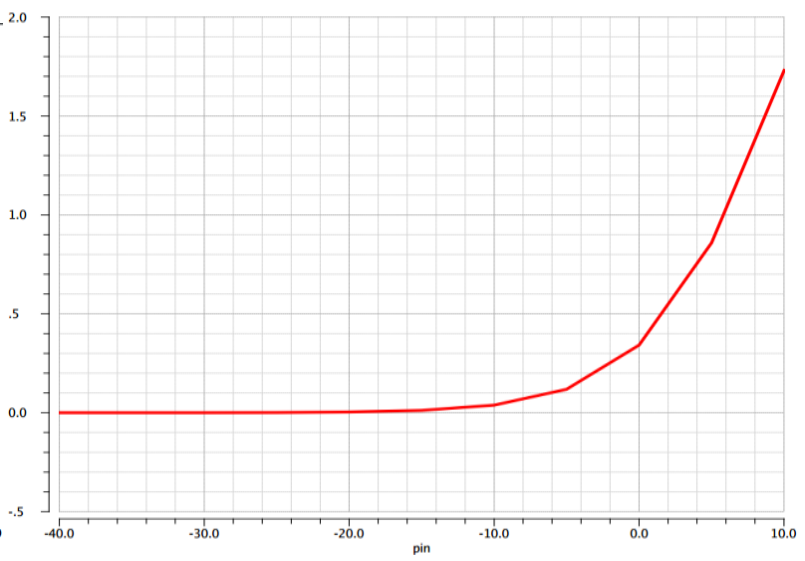

(b)

Fig. 8: (a) Kf (b) PAE.

\section{Conclusion}

This paper describes the method of designing and simulating power amplifier using cadence software based on SIMC CMOS process $180 \mathrm{~nm}$ technology. This PA is used for sensor networks. This research is still in the early stages of development of a low cost and low power device. In order to reach the performance that is needed, the PA process uses group III and IV elements. This circuit meets the scheduled requirements for the CMOS process, but it still has room to improve performance metrics. When the sensor is coupled with communications technologies such as mobile phones and the Internet, the sensor network constant information flow between individuals and their doctors. Such low cost and low power device can save a lot of hospitalization resources. To realize this, future improvement is needed.

\section{References}

[1] B.M. Stults, "Preventive Health Care for the Elderly," Western Journal of Medicine, pp. 832-845, 1984.

[2] A. Minaie, A. Sanati-Mehrizy, P. Sanati-Mehrizy, R. Sanati-Mehrizy, "Application of Wireless Sensor Networks in Health Care System," ASEE, pp. 21-24, 2013.

[3] J. Gong et al., "Wafer edge defect study of temporary bonded and thin wafers in TSV process flow," in Proceedings of the 2015 IEEE 65 ${ }^{\text {th }}$ Electronic Components and Technology Conference (ECTC), San Diego, CA, 2015, pp. 17071712 .

[4] J. Gong, P. Vukkadala, J. K. Sinha, and K. T. Turner, "Determining local residual stresses from high resolution wafer geometry measurements" Journal of Vacuum Science \& Technology B, vol. 31, pp. 051205, 2013.

[5] J. Gong and I. C. Ume, "Nondestructive Evaluation of Poor-Wetted Lead-Free Solder Bumps in Ball Grid Array Packages Using Laser Ultrasound and Interferometric Technique," IEEE Transactions on Components, Packaging and Manufacturing Technology, vol. 3, no. 8, pp. 1301-1309, 2013. 
[6] I. C. Ume and J. Gong, "Evaluation of Lead-Free Solder Bump Voiding Ball Grid Array Packages Using Laser Ultrasound and Interferometric Technique," IEEE Transactions on Components, Packaging and Manufacturing Technology, vol. 3, no. 8, pp. 1310-1320, 2013.

[7] W. C. Frank Shi, "2.4 GHz Heterodyne Receiver for Healthcare Application," International Journal of Pharmacy and Pharmaceutical Sciences, vol. 8, no. 6, 2016.

[8] W. cai, L. Huang, W. Wen, "2.4GHZ Class AB Power Amplifier for Healthcare Application," International Journal of Biomedical Engineering and Science (IJBES), vol. 3, no. 2, 2016.

[9] W. Cai, L. Huang, W.J. Wen, "2.4GHZ Class AB Power Amplifier for Healthcare Application," International Journal of Biomedical Engineering and Science (IJBES), vol. 3, no. 2, 2016.

[10] W. Cai, L. Huang, W.J. Wen "2.4GHZ Class AB Power Amplifier for Wireless Medical Sensor Network," International Journal of Enhanced Research in Science, Technology \& Engineering, vol. 5, no. 4, 2016.

[11] L. Cheng, P. Li, T. T. Mo, “A 1.9 GHZ High Efficiency Class-F SOI CMOS Power,” CISIA, pp. 347-349, 2015.

[12] L. Junming, Z. Guohao, Z. Yaohua, L. Sizhen, Z. Zhihao, C. Sidi, "Design of broadband class-F power amplifier with high-order harmonic suppression for S-band application,” J of Semi, pp. 125002-1-5, 2015. 